\section{Trasplante de progenitores hematopoyéticos en un paciente con enfermedad granulomatosa crónica en México}

Nideshda Ramírez-Uribe ${ }^{1}$, Claudia Hernández-Martínez², Gerardo LópezHernández ${ }^{1}$, Martín Pérez-García ${ }^{1}$, Emmanuel Ramírez-Sánchez ${ }^{4}$, Sara Elva Espinosa-Padilla ${ }^{2}$, Marco Yamazaki-Nakashimada ${ }^{3}$, Alberto Olaya-Vargas ${ }^{1}$, Lizbeth Blancas-Galicia²

\section{Resumen}

La incidencia de la enfermedad granulomatosa crónica en reportes internacionales es de 1:250,000; sin embargo, en México se desconoce. En el Instituto Nacional de Pediatría, a partir de 2009 se implementó un proyecto para facilitar el diagnóstico de esa enfermedad. De esa fecha al día de hoy se han estudiado 68 casos, de los que $80 \%$ son formas ligadas al cromosoma $X$, además de que cada vez los casos se han diagnosticado a una edad más temprana. En la actualidad nos encontramos con un nuevo reto: el tratamiento curativo de los pacientes con enfermedad granulomatosa crónica. Comunicamos el caso de un paciente con enfermedad granulomatosa crónica ligada al cromosoma $\mathrm{X}$, trasplantado exitosamente al mes de vida en México.

PALABRAS CLAVE: trasplante, progenitores hematopoyéticos, enfermedad granulomatosa crónica.

Rev Alerg Méx 2016 Jan-Mar;63(1):95-103.

\section{Hematopoietic progenitors transplantation in a patient with chronic granulomatous disease in Mexico.}

Nideshda Ramírez-Uribe ${ }^{1}$, Claudia Hernández-Martínez², Gerardo LópezHernández ${ }^{1}$, Martín Pérez-García ${ }^{1}$, Emmanuel Ramírez-Sánchez ${ }^{4}$, Sara Elva Espinosa-Padilla ${ }^{2}$, Marco Yamazaki-Nakashimada ${ }^{3}$, Alberto Olaya-Vargas ${ }^{1}$, Lizbeth Blancas-Galicia ${ }^{2}$

\begin{abstract}
The incidence of chronic granulomatous disease in international reports is 1:250,000; however, in Mexico it is unknown. At the National Institute of Pediatrics of Mexico a project for facilitating the diagnosis of the disease was implemented by us in 2009. From the start of such project up to date 68 cases have been studied; $80 \%$ of those are X-linked forms (LX) and moreover, it has become noticeable the diagnosis at a younger age. The new challenge we are facing its to provide a successful treatment to those patients diagnosed with chronic granulomatous disease (CGD). We are reporting the case of a one-month old newborn patient diagnosed with CGD-LX that was successfully transplanted in Mexico.
\end{abstract}

KEYWORDS: transplant; hematopoietic progenitors; chronic granulomatous disease
1 Unidad de Trasplante de Células Progenitoras Hematopoyéticas.

${ }^{2}$ Unidad de Investigación en Inmunodeficiencias. ${ }^{3}$ Servicio de Inmunología.

Instituto Nacional de Pediatría, Secretaría de Salud, Ciudad de México.

${ }^{4}$ Inmunología, Hospital General Playa del Carmen, Secretaría de Salud, Playa del Carmen, Quintana Roo, México.

Recibido: 3 de septiembre 2015

Aceptado: 14 de diciembre 2015

Correspondencia

Dra. Lizbeth Blancas Galicia

blancas.lizbeth@gmail.com

Este artículo debe citarse como

Ramírez-Uribe N, Hernández-Martínez $\mathrm{C}$, LópezHernández G, Pérez-García M y col. Trasplante de progenitores hematopoyéticos en un paciente con enfermedad granulomatosa crónica en México. Rev Alerg Méx. 2016 ene-mar;63(1):95-103. 


\section{ANTECEDENTES}

La enfermedad granulomatosa crónica es una inmunodeficiencia primaria causada por un defecto en el estallido respiratorio de los fagocitos (neutrófilos, eosinófilos, monocitos y macrófagos), que juega un papel crítico en la muerte de bacterias y hongos. La enzima que cataliza el estallido respiratorio es la NADPH oxidasa, que consiste en un complejo enzimático formado por seis subunidades (gp91phox, p22phox, p47phox, p67phox, p40phox, Rac2). El gen que codifica para la subunidad gp91 phox es $C Y B B$, se encuentra en el cromosoma $\mathrm{X}$ y el resto de los genes CYBA, NCF1, NCF2, NCF4 y $R A C 2$ están en los autosomas. ${ }^{1-3}$

Como resultado del defecto de la NAPDP-oxidasa la mayoría de los pacientes con enfermedad granulomatosa crónica sufren infecciones graves y recurrentes, además, carecen de regulación en el proceso inflamatorio; secundariamente padecen granulomas difusos que pueden llegar a causar síntomas como obstrucción en el esófago, el estómago, los uréteres, la vejiga urinaria o trastornos disfuncionales secundarios a fibrosis extensa de los diferentes sistemas. Uno de los primeros signos de la enfermedad en los que reciben la vacuna de BCG es BCGitis.

El diagnóstico de la enfermedad granulomatosa crónica se hace al demostrar ausencia de la producción de radicales libres en los neutrófilos activados, mediante diferentes pruebas, como reducción de nitroazul de tetrazolio, reducción de ferrocitocromo o 1,2,3 dihidrorodamina. El estado de portador en la forma recesiva ligada al cromosoma $X$ puede detectarse a través de 1,2,3 dihidrorodamina. ${ }^{3,4}$

La mayoría de los pacientes con enfermedad granulomatosa crónica son tratados a largo plazo con profilaxis antimicrobiana y antifúngica, con lo que disminuye el riesgo de padecer infecciones fatales, mas no desaparece. La administración de interferón-gamma recombinante es motivo de controversia. Por tanto, el trasplante de células progenitoras hematopoyéticas es el único tratamiento potencialemente curativo en la actualidad. ${ }^{5}$

Entre los dos patrones de trasmisión hereditaria la forma recesiva ligada al cromosoma $\mathrm{X}$ es la más frecuente (60-80\%) en comparación con la forma autosómica recesiva. ${ }^{2,3,5}$

La incidencia de la enfermedad granulomatosa crónica en reportes internacionales es de 1:250,000; sin embargo, en México se desconoce. En el Instituto Nacional de Pediatría a partir de 2009 se implementó un proyecto para facilitar el diagnóstico de enfermedad granulomatosa crónica y el tipo de herencia, a través de la prueba de 1,2,3 dihidrorodamina. ${ }^{6}$

Debido a que la enfermedad granulomatosa crónica recesiva ligada al cromosoma $X$ es fatal y el único tratamiento potencialmente curativo es el trasplante de células progenitoras hematopoyéticas, el Instituto Nacional de Pediatría (centro nacional de referencia de inmunodeficiencias primarias), ya lo realiza en pacientes con esta enfermedad. Comunicamos el caso de un paciente con diagnóstico temprano de enfermedad granulomatosa crónica recesiva ligada al cromosoma $\mathrm{X}$ en el primer mes de vida y trasplantado exitosamente antes del año de edad.

\section{CASO CLÍNICO}

Paciente masculino de un año tres meses de edad al momento del reporte, originario de Oaxaca, México, con antecedente familiar de padres no consanguíneos, producto del quinto embarazo, tres hermanos varones con antecedente de fiebre, candidiasis oral de repetición, BCGitis, otitis, hiporexia y pérdida de peso, todos finados antes de los dos años de edad. El cuarto herma- 
no tenía antecedente de BCGosis, neumonía y candidiasis oral (Figura 1).

En el caso que comunicamos hubo varios datos que orientaron el diagnóstico de una inmunodeficiencia primaria, específicamente de enfermedad granulomatosa crónica, como el antecedente de varios varones afectados en la familia, finados por infecciones recurrentes y severas. Realizamos como prueba de tamizaje $1,2,3$ dihidrorodamina en el cuarto hermano sintomático y en el entonces recién nacido asintomático.

Los resultados mostraron falta de producción de radicales libres en los dos varones. Por tanto, realizamos la misma prueba en la madre y la hija con el fin de detectar si eran portadoras de enfermedad granulomatosa crónica recesiva ligada al cromosoma X; sólo la madre mostró un patrón bimodal compatible con el estado de portador (Figura 2). La hermana mostró un patrón normal similar al control.
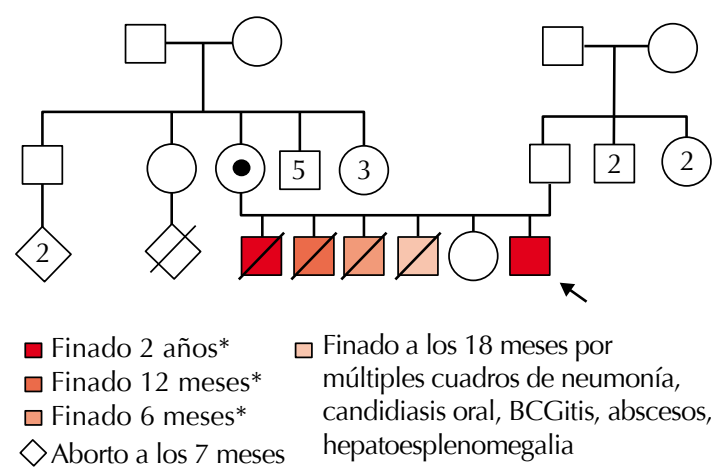

* Padecieron complicaciones infecciosas que en ocasiones ameritaron hospitalizaciones, como cuadros febriles, candidiasis oral, BCGitisy otitis.

Figura 1. Árbol genealógico que muestra los antecedentes de hermanos varones finados a edades tempranas; todos padecieron infecciones recurrentes. La madre es portadora.
A los nuevos casos con enfermedad granulomatosa crónica se les cuantificó la expresión de gp91phox por citometría de flujo; ambos mostraron nula expresión, lo que se asocia con mal pronóstico.

Un mes después del diagnóstico el cuarto hermano sintomático falleció debido a sepsis con foco pulmonar. En el otro caso se inició inmediatamente profilaxis antimicrobiana, tratamiento con interferón-gamma, no se vacunó con BCG y fue referido al tercer nivel de atención médica.

Posterior a la evaluación médica se decidió realizar trasplante de células progenitoras hematopoyéticas, tomando en cuenta que se trataba de un caso con un fenotipo severo, de acuerdo con:

1. Patrón de herencia de enfermedad granulomatosa crónica recesiva ligada al cromosoma $\mathrm{X}$.

2. Nula expresión de gp91phox por citometría de flujo.

3. Un hermano finado por enfermedad granulomatosa crónica y tres hermanos finados con el mismo cuadro clínico, pero sin diagnóstico.

Se seleccionó a la hermana sana de cinco años de edad como donadora por ser emparentada con HLA 10/10 (HLA-A, HLA-B, HLA-C, HLA-DRB1, HLA-DQA1, HLA-DQB1). Como parte del protocolo se valoraron los diferentes sistemas; en evacuaciones se aisló Clostridium difficile, que remitió con metronidazol. A los 10 meses de edad del paciente se realizó el trasplante de células progenitoras hematopoyéticas, que se obtuvieron de médula ósea, se usó un régimen de acondicionamiento mieloablativo con busulfán 16 mg/kg/día, ciclofosfamida 120 $\mathrm{mg} / \mathrm{kg} /$ día y gammaglobulina antitimocito 4.5 $\mathrm{mg} / \mathrm{kg} /$ día (día -11). La dosis infundida de células progenitoras hematopoyéticas fue de CD34+ $5.22 \times 10^{6}$ y mononucleares $8.5 \times 10^{7}$ (día 0 ). 


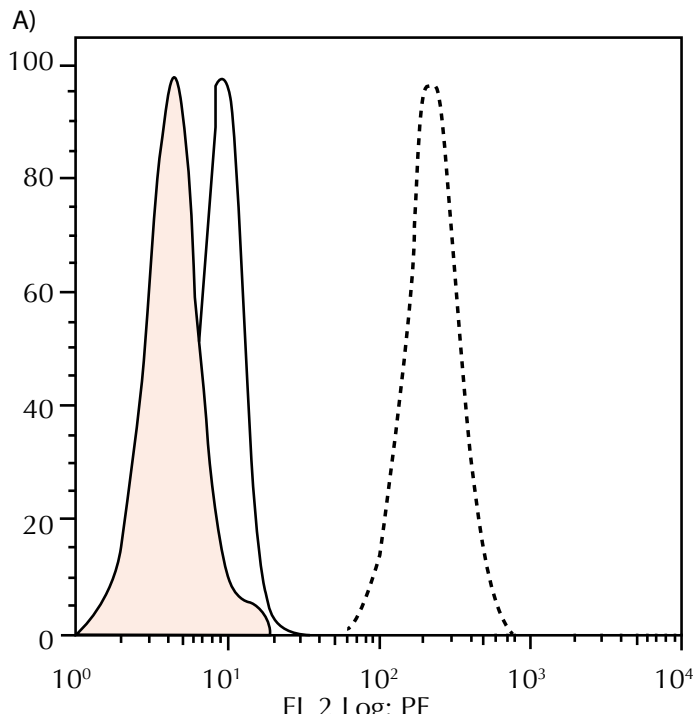

FI 2 log, SS Lin subset Testigo

C)

Índice de oxidación de 26

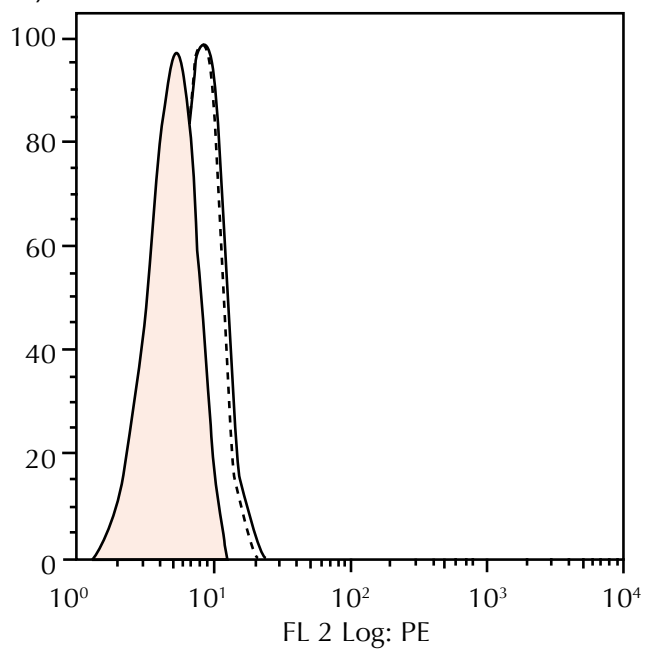

FL 2 Log, SS Lin subset Paciente recién nacido Índice de oxidación 1

Producción de radicales libres

B)

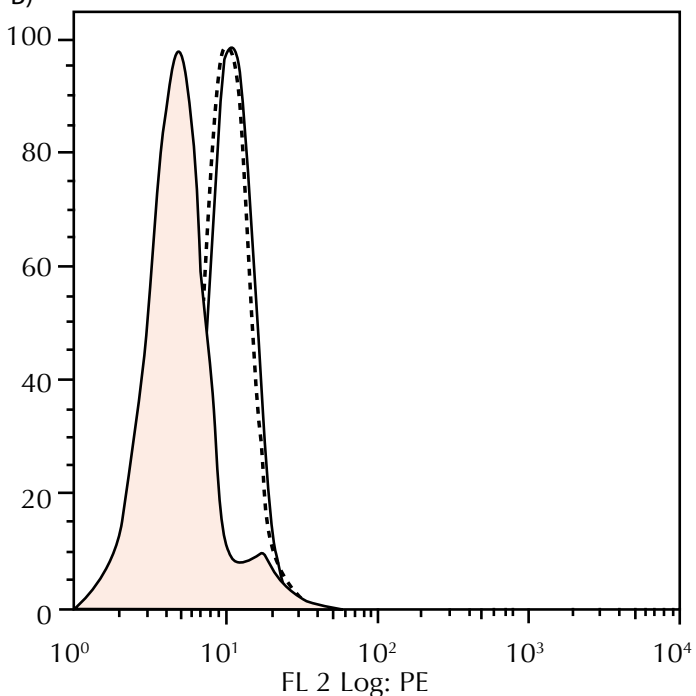

FL 2 log, ss Lin subset

Paciente (hermano 4)

D)

Índice de oxidación de 1

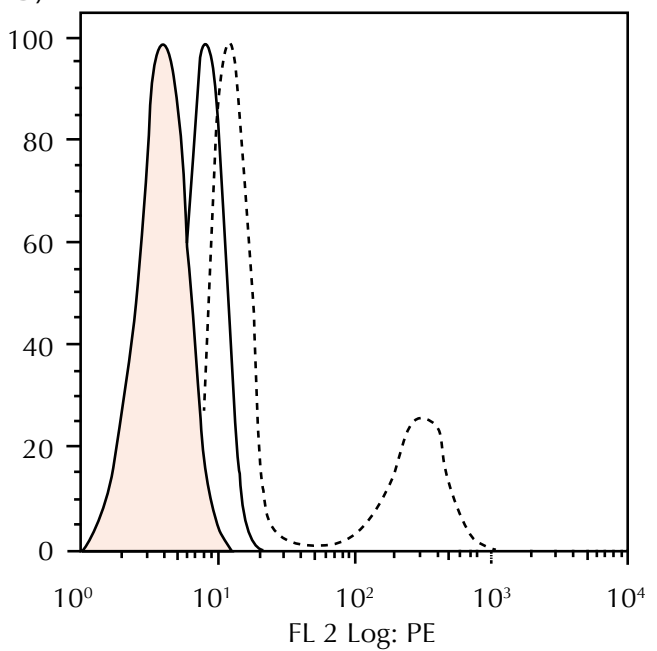

FS Lin, SS Lin subset

Madre

Primer pico con índice de oxidación 1.4

Segundo pico con índice de oxidación 35.5

Figura 2. Ensayo de 1,2,3 dihidrorodamina. A. Producción normal de radicales libres en neutrófilos representada por el histograma punteado dezplazado a la derecha sobre el eje de las X. B y C. Falta de producción de radicales libres en los dos hermanos con enfermedad granulomatosa crónica; el histograma punteado no tiene un desplazamiento hacia la derecha. D. Estado de portadora de la madre; el histograma punteado tiene un patrón bimodal que representa las dos poblaciones de neutrófilos en la madre del paciente, la de la izquierda con produción anormal (índice de oxidación de 1.4) y la de la derecha con producción normal (índice de oxidación de 35). 
Al día +13 hubo evidencia de recuperación en la cuenta absoluta de neutrófilos (más de $500 \mathrm{cel} /$ $\mathrm{mm}^{3}$ durante tres días sostenidos) y quimerismo del $100 \%$. Al día +14 del trasplante se aisló adenovirus en evacuaciones; sin embargo, el paciente se mantuvo asintomático. Al día +33 tuvo enfermedad de injerto contra huésped en piel grado II, que se trató exitosamente con tacrolimus tópico y metilprednisolona a $1 \mathrm{mg} / \mathrm{kg} /$ día. En el día +50 postrasplante se documentó infección por citomegalovirus, que se negativizó al día +58; en el día +106 se documentaron adenopatías cervicales derechas, la biopsia por escisión no mostró anormalidades y las cargas virales en ese momento para citomegalovirus, virus de Epstein-Barr y adenovirus tuvieron reportes negativos. El último quimerismo del día +92 fue del $100 \%$. Al día +118 en muestra de sangre periférica se evaluó el estallido respiratorio por ensayo de 1,2,3 dihidrorodamina y la expresión de la proteína gp91phox en neutrófilos (citometría de flujo, en comparación con un sujeto sano); observamos que en relación con los estudios previos al trasplante de células progenitoras hematopoyéticas hubo mejoría en ambos resultados (Figura 3). En la actualidad, el paciente tiene seguimiento en la consulta externa y se ha mantenido en buenas condiciones, sin eventos infecciosos o inflamatorios, continúa con tratamiento antimicrobiano profiláctico, este último se indicó por los efectos de mieloablación del acondicionamiento (Figura 4).

\section{DISCUSIÓN}

No existen reportes epidemiológicos de la enfermedad granulomatosa crónica en México; en 2002 se publicó un registro de las inmunodeficiencias primarias de 30 años del Instituto Nacional de Pediatría, se recabaron 171 casos y de ellos sólo 12 correspondieron a enfermedad granulomatosa crónica. En la Unidad de Investigación en Inmunodeficiencias, de 2009 a 2015 estudiamos 68 casos, documentamos que el primer síntoma de la enfermedad en

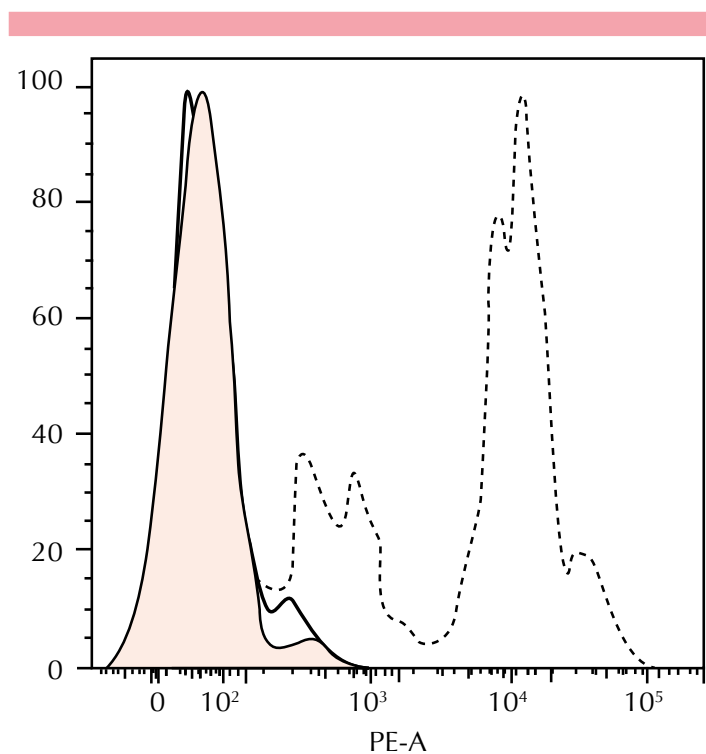

FSC-A, SSC-A subset

Paciente posterior al trasplante de células progenitoras hematopoyéticas Primer pico 6.3

Segundo pico 81

Figura 3. Ensayo de 1,2,3 dihidrorodamina. Se observa la producción de radicales libres en neutrófilos, representada por el histograma punteado desplazado a la derecha sobre el eje de las X. Observamos que hay un patrón bimodal en el paciente, lo que refleja que tiene dos poblaciones con diferentes índices de oxidación, que corresponden a las células del receptor (izquierda) y a las del donador (derecha).

México puede ser una reacción adversa local a la vacuna de BCG, al igual que en otros países de Latinoamérica. ${ }^{7}$ En países en los que no se aplica la vacuna de BCG, en general de primer mundo, no se observan los efectos adversos de la vacuna de BCG como parte de la enfermedad granulomatosa crónica. Ante un evento de BCGitis, BCGosis, otro foco infeccioso o los tres, debe incluirse a la enfermedad granulomatosa crónica como parte del diagnóstico diferencial. En los 68 casos otras manifestaciones frecuentes fueron neumonías, adenitis, sepsis o fiebre sin foco infeccioso aparente. ${ }^{6}$ 


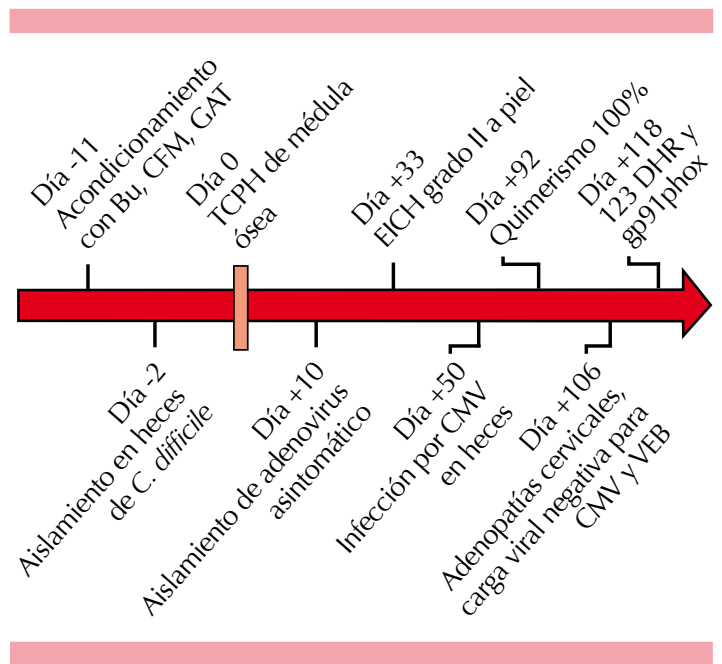

Figura 4. Diferentes eventos alrededor del trasplante de células progenitoras hematopoyéticas (TCPH). Bu: busulfán; CMF: ciclofosfamida; GAT: gammaglobulina antitimocito; 1,2,3 DHR: ensayo de dihidrorodamina; gp91phox: cuantificación de la expresión de la proteína posterior al trasplante; CMV: citomegalovirus; VEB: virus de Epstein-Barr.

Los pacientes con enfermedad granulomatosa crónica con herencia recesiva ligada al cromosoma $\mathrm{X}$ tienen menor supervivencia y mayor mortalidad. En un estudio europeo de 429 pacientes con enfermedad granulomatosa crónica se describió, al momento del estudio, un promedio de vida de 49.6 años para pacientes con enfermedad granulomatosa crónica autosómica y de 37.8 años para pacientes con enfermedad granulomatosa crónica recesiva ligada al cromosoma $X$; la supervivencia a 10 años en el primer grupo fue de $94 \%$ y de $86 \%$ para el segundo. Se ha reportado que tan sólo $50 \%$ de los pacientes alcanzan los 30 años, a pesar de llevar apego estricto al tratamiento profiláctico; $30 \%$ de los pacientes muere por aspergilosis. EI trasplante de células progenitoras hematopoyéticas es una alternativa terapéutica con potencial de curar la enfermedad granulomatosa crónica y de revertir el daño a órganos blanco. ${ }^{5,8-10}$

Existe controversia de cuándo es el momento ideal para realizar el trasplante de células progenitoras hematopoyéticas en la enfermedad granulomatosa crónica. Los pacientes con ausencia de la actividad de la NADPHoxidasa tienen mal pronóstico, por lo que se recomienda que en ellos el trasplante se realice en edades tempranas. Los pacientes con enfermedad granulomatosa crónica recesiva ligada al cromosoma $X$, en general, tienen actividad nula de la NADPH-oxidasa. Según Gungor y su grupo, las indicaciones generales para realizar el trasplante de células progenitoras hematopoyéticas son:

1. Una o más infecciones que pongan en riesgo la vida del paciente.

2. Falta de apego al tratamiento profiláctico antimicrobiano.

3. Dependencia de esteroides por enfermedad autoinflamatoria.

El inciso 1 incluye a los pacientes con infecciones activas, como aspergilosis, que no responden al tratamiento y en los que el trasplante de células progenitoras hematopoyéticas es la única alternativa de curación. Asimismo, en pacientes adolescentes y en adultos jóvenes, estos criterios son más difíciles de aplicar debido a que en ellos ya existe daño a diferentes órganos y la mortalidad después del trasplante es alta. ${ }^{8,11}$

Diversos autores demostraron disminución de la morbilidad y mortalidad en pacientes con enfermedad granulomatosa crónica. Cole y colaboradores compararon un grupo de 32 pacientes con enfermedad granulomatosa crónica: casos antes de ser trasplantados y casos no trasplantados contra casos trasplantados; el último grupo tuvo, por mucho, menor número de admisiones hospitalarias por infecciones, colitis, colonoscopia y cirugías, y mejoría en la curva de crecimiento. Cole y su grupo, además, consideran que el éxito del trasplante de manera global se asocia con la óptima selección de un donador relacionado, la administración de fármacos 
menos tóxicos durante el acondicionamiento y de antivirales cada vez mejores. ${ }^{12}$

El proceso de trasplante de células progenitoras hematopoyéticas en sí mismo tiene limitaciones, como disponibilidad de donantes, riesgos asociados con el esquema de acondicionamiento y las complicaciones que surjan de la interacción inmunitaria entre el huésped y el donante. ${ }^{13}$

Diversos centros hospitalarios reportaron su experiencia en el trasplante de células progenitoras hematopoyéticas. En estos estudios se describen los diversos regímenes usados, inicialmente con un acondicionamiento mieloablativo y en un estudio reciente con esquema de acondicionamiento de intensidad reducida. La dosis de mieloablación óptima para generar espacio en los nichos receptores e inmunosuprimir al huésped, sin tener sus efectos adversos como enfermedad de injerto contra huésped e infecciones fatales, es todo un reto.

Soncini y su grupo describieron los resultados de 20 pacientes trasplantados en el periodo de 1998 a 2007, los pacientes tenían entre 15 meses y 21 años de edad; el trasplante en 10 de ellos fue con donantes compatibles de hermanos, nueve con médula ósea y uno con sangre del cordón umbilical. El resto recibió trasplantes de donador no relacionado con HLA compatible. El seguimiento varió de 4 a 117 meses, 18 pacientes sobrevivieron con una función normal de neutrófilos $(90 \%)$ y dos murieron a causa de infecciones por hongos. ${ }^{10}$

En Reino Unido e Irlanda se evaluaron 94 pacientes con enfermedad granulomatosa crónica en el año 2000. Los resultados mostraron una función casi normal de los neutrófilos (90\%) después del trasplante de células progenitoras hematopoyéticas. Además, este estudio fue el primero que demostró que la calidad de vida es adecuada después del trasplante de células progenitoras hematopoyéticas; todos los casos tuvieron un efecto positivo en el desarrollo de su capacidad emocional, social y escolar en la vida cotidiana a largo plazo. ${ }^{14,15}$

En la enfermedad granulomatosa crónica se han descrito regímenes de acondicionamiento reducido en pacientes con mayor riesgo, como son pacientes adolescentes o adultos que no pueden tolerar regímenes mieloablativos por infecciones activas (demostradas por microbiología o radiología) o autoinflamación, que no remite al tratamiento, con fiebre de $38^{\circ} \mathrm{C}$ o proteína $\mathrm{C}$ reactiva elevada (o ambas). Este grupo etario tiene mortalidad asociada con el trasplante de 28 a 50\%. Estos regímenes de acondicionamiento reducido tienen como objetivo incrementar el injerto mieloide y reducir la toxicidad a órganos. ${ }^{8}$ Gungor y su grupo incluyeron a 56 pacientes con los criterios enumerados previamente de 16 centros hospitalarios de 10 países, durante el periodo de 2003 a 2012, la edad promedio de los pacientes fue de 12.7 años; 21 tenían donador relacionado con HLA compatible y 35 donador no relacionado con HLA 9/10 o 10/10; la fuente de las células progenitoras hematopoyéticas fue la médula ósea en 45 pacientes y sangre periférica en 11 . El régimen de acondicionamiento incluyó fludarabina y busulfán, en algunos casos se adicionaron dosis bajas de globulina antitimocito. El injerto ocurrió, en promedio, a los 19 días. En una mediana de seguimiento de 21 meses, la supervivencia global fue de 93\% (52 de 56), la probabilidad de supervivencia global a dos años fue de $96 \%$ y de supervivencia libre de eventos fue de $91 \%$. La incidencia acumulada de enfermedad injerto contra huésped grave (grado III-IV) fue sólo de $4 \%$ y la forma crónica representó 7\%. El 93\% de los pacientes sobrevivió. ${ }^{8}$

No debemos pasar por alto las complicaciones propias de la enfermedad granulomatosa crónica posterior a trasplante, como la colitis inflamatoria y la enfermedad inflamatoria progresiva 
del pulmón, ambas como parte de una enzima $\mathrm{NPDH}$-oxidasa alterada en la enfermedad granulomatosa crónica y que regula anormalmente el proceso de inflamación. ${ }^{8}$

El estado del injerto en enfermedad granulomatosa crónica puede evaluarse con herramientas como la determinación de quimerismo; sin embargo, otras técnicas útiles son la comparación de la 1,2,3 dihidrorodamina (evalúa el estallido respiratorio) y la expresión positiva de la proteína gp91 phox en neutrófilos, monocitos o ambos, antes y después del trasplante. Ambas muestran la existencia de una población celular (receptor $100 \%$ o donador $100 \%$ ) o dos poblaciones celulares (cierto porcentaje del paciente y cierto porcentaje del donador). En la enfermedad granulomatosa crónica se requiere un mínimo de $10 \%$ de las células del donador para que el paciente esté libre de manifestaciones clínicas. Otras ventajas de estas herramientas son su simplicidad, rapidez y costo-efectividad. ${ }^{16,17}$

Por último, la terapia génica tendría varias ventajas teóricas sobre el trasplante de células progenitoras hematopoyéticas, incluida la pronta disponibilidad de células madre hematopoyéticas propias del paciente, sin riesgo de enfermedad injerto contra huésped. Sin embargo, la falta sostenida del gen marcado en el compartimiento mieloide en los primeros ensayos resulta en una corrección funcional insignificante de la enfermedad y graves eventos negativos (mielodisplasia y leucemia mieloide aguda) causados por mutagénesis de inserción. En México aún no se cuenta con terapia génica; sin embargo, parece ofrecer una alternativa prometedora en el futuro que incluso podría sustituir al trasplante de células progenitoras hematopoyéticas. ${ }^{18}$

La difusión de las inmunodeficiencias primarias en México ha llevado a su mayor diagnóstico, incluida la enfermedad granulomatosa crónica (fumeni.org.mx). El reto al que nos enfrentamos ahora es ofrecer un tratamiento curativo a los nuevos casos. Una de las limitantes en México es la falta de suficientes donadores de células progenitoras hematopoyéticas. En nuestro país la Fundación Comparte Vida A.C. tiene entre sus objetivos fomentar la cultura de la donación mediante la realización de campañas diversas de difusión y educación continua para trasmitir a la población la importancia de convertirse en un donador potencial altruista al apoyar a los miles de pacientes que requieren un trasplante de médula ósea.

En el caso que comunicamos, la edad temprana al momento del diagnóstico y el tratamiento curativo son factores que reflejan el esfuerzo de un equipo multidisciplinario que ha trabajado en la difusión de las inmunodeficiencias primarias en los médicos de primer contacto. En la actualidad, la Unidad de Investigación en Inmunodeficiencias (UIID) forma parte de una red de diagnóstico a nivel nacional, integrada por diferentes inmunólogos (http://fumeni.org. mx/encuentra-un-inmunologo/), que después de una valoración clínica del paciente con infecciones severas y recurrentes, compatibles con enfermedad granulomatosa crónica, nos envían las muestras para realizar pruebas de tamizaje. Los casos diagnosticados se refieren inmediatamente a un grupo de médicos, entre ellos, médicos especialistas en la realización de trasplante de células progenitoras hematopoyéticas.

\section{Agradecimientos}

Lizbeth Blancas Galicia y Sara Espinosa Padilla forman parte del Sistema Nacional de Investigadores. El proyecto fue financiado por CONACYT: SALUD-2012-01-180910 y por la Fundación Mexicana para Niñas y Niños con Inmunodeficiencias. 


\section{REFERENCIAS}

1. Roos D, et al. Hematologically important mutations: the autosomal recessive forms of chronic granulomatous disease (second update). Blood Cells Mol Dis 2010;44:291-299.

2. Rosenzweig SD. Inflammatory manifestations in chronic granulomatous disease (CGD). J Clin Immunol 2008;28(Suppl 1):S67-72.

3. Winkelstein JA, et al. Chronic granulomatous disease. Report on a national registry of 368 patients. Medicine (Baltimore) 2000;79:155-169.

4. Berron-Ruiz $L$, et al. Detection of inheritance pattern in thirty-three Mexican males with chronic granulomatous disease through 123 dihydrorhodamine assay. Allergol Immunopathol (Madr) 2014;42:580-585.

5. van den Berg JM, et al. Chronic granulomatous disease: the European experience. PLoS One 2009;4:5234.

6. González A. Características clinicas y moleculares de pacientes mexicanos con enfermedad granulomatosa crónica diagnosticados durante los últimos 10 años. 2015, Facultad de Medicina. División de estudios de posgrado e investigación UNAM, 1-44.

7. de Oliveira-Junior EB, et al. Clinical and genotypic spectrum of chronic granulomatous disease in 71 Latin American patients: First report from the LASID registry. Pediatr Blood Cancer 2015.

8. Gungor T, et al. Reduced-intensity conditioning and HLA-matched haemopoietic stem-cell transplantation in patients with chronic granulomatous disease: a prospective multicentre study. Lancet 2014;383:436-448.

9. Martinez CA, et al. Excellent survival after sibling or unrelated donor stem cell transplantation for chronic granulomatous disease. J Allergy Clin Immunol 2012;129:176183.
10. Soncini E, et al. Unrelated donor and HLA-identical sibling haematopoietic stem cell transplantation cure chronic granulomatous disease with good long-term outcome and growth. Br J Haematol 2009;145:73-83.

11. Klaudel-Dreszler MA, et al. Treosulfan-based conditioning regimen in a second matched unrelated peripheral blood stem cell transplantation for a pediatric patient with CGD and invasive aspergillosis, who experienced initial graft failure after RIC. Int J Hematol 2009;90:571-575.

12. Cole $\mathrm{T}$, et al. Clinical outcome in children with chronic granulomatous disease managed conservatively or with hematopoietic stem cell transplantation. J Allergy Clin Immunol 2013;132:1150-1155.

13. Prasad VK. Stem-cell transplantation for chronic granulomatous disease. Lancet 2014;383:390-392.

14. Cole $T$, et al. Health related quality of life and emotional health in children with chronic granulomatous disease: a comparison of those managed conservatively with those that have undergone haematopoietic stem cell transplant. J Clin Immunol 2013;33:8-13.

15. Jones LB, et al. Special article: chronic granulomatous disease in the United Kingdom and Ireland: a comprehensive national patient-based registry. Clin Exp Immunol 2008;152:211-218.

16. Kim HY, et al. Rapid determination of chimerism status using dihydrorhodamine assay in a patient with $\mathrm{X}$-linked chronic granulomatous disease following hematopoietic stem cell transplantation. Ann Lab Med 2013;33:288-292.

17. Gonzalez-Ortiz A. Características clínicas y moleculares de pacientes mexicanos con enfermedad granulomatosa crónica diagnosticados durante los últimos 10 años. División de Posgrado e Investigación. México: UNAM, 2015.

18. Notarangelo LD. The long road to optimal management for chronic granulomatous disease. J Allergy Clin Immunol 2013;132:1164-1165. 